\title{
Radio Observations of Mercury, Venus, Mars, Saturn, and Uranus
}

\author{
Kenneth I. Kellermann ${ }^{1}$ \\ CSIRO Radiophysics Laboratory, Sydney, Australia
}

This paper summarizes the results of observations of radio emission from Mercury, Venus, Mars, Saturn, and Uranus made with the CSIRO $210-\mathrm{ft}$ radio telescope at Parkes. A description of the main results for each planet is given here. A more detailed discussion will be published elsewhere.

Mercury-Observations of the $11-\mathrm{cm}$ thermal radiation from Mercury made in 1964 showed that the temperature of the night side is considerably greater than was expected if Mercury was a smooth nonrotating planet having a low thermal conductivity and no atmosphere [Kellermann, 1965]. These temperatures, together with some more recent values are shown in figure 1. The measurements made at large phase angles, which refer primarily to the nighttime side of the planet, indicate a temperature of about $250{ }^{\circ} \mathrm{K}$. The temperature on the daytime side is less well determined but seems to be somewhat less than the $510{ }^{\circ} \mathrm{K}$ expected from infrared measurements [Pettit, 1961]. The high temperature found on the night side can be explained if either there is a thin atmosphere which convects heat from the day to nighttime side of the planet, or if the planet is not in synchronous rotation, and both sides are heated by solar radiation.

\footnotetext{
${ }^{1}$ Present address: National Radio Astronomy Observatory, Green Bank, W. Va.
}

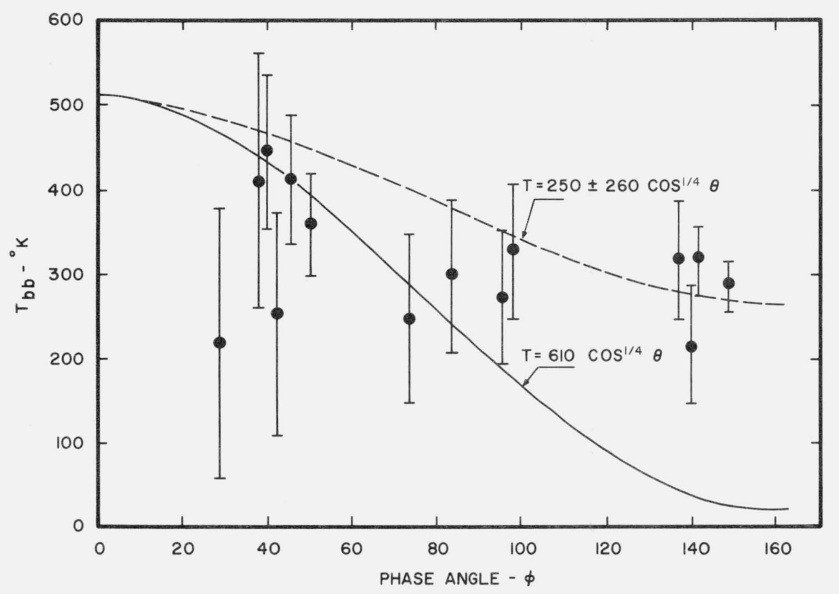

FigURE 1. Observed blackbody temperature for Mercury as a function of planetocentric phase angle, $\phi$.

The solid curve represents the expected phase law if the dark side is at zero degrees and the temperature of the illuminated side is equal to $610 \cos ^{1 / 4} \theta$ where $\theta$ is the angle between any point on the surface and the subsolar point as seen from the center of the planet. The dashed curve corresponds to a uniform temperature of $250^{\circ} \mathrm{K}$ over the whole surface plus $260 \cos ^{1 / 4} \theta$ for $\theta<90^{\circ}$ (illuminated hemisphere).
However, the low radio temperature found on the daytime side cannot be explained by convection, but can be understood if the planet's rotation period of $59 \pm 5$ days [Pettengill and Dyce, 1965] is shorter than the thermal time constant at depths below Mercury's surface where the thermal radio emission originates.

Venus - The radiation from Venus was measured at four wavelengths near the inferior conjunction of June 1964. The results, which are shown in table 1, indicate a decrease in the equivalent blackbody temperature toward the longer wavelengths. This may be due to the increased radio reflectivity of the planet at decimeter wavelengths. Observations made near superior conjunction at $11 \mathrm{~cm}$ show that any variation in the temperature with phase angle is small at this frequency.

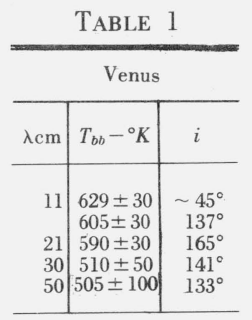

Mars-Observations of Mars made near the unfavorable opposition of March 1965 indicate an equivalent blackbody temperature of slightly under $200^{\circ} \mathrm{K}$ (see table 2) at 6,11 , and $21 \mathrm{~cm}$. This may be compared with the infrared temperature of about $250{ }^{\circ} \mathrm{K}$ and the $3 \mathrm{~cm}$ temperature of $211{ }^{\circ} \mathrm{K}$ measured at the Naval Research Laboratory [Giordmaine, Alsop, Townes, and Mayer; 1959].

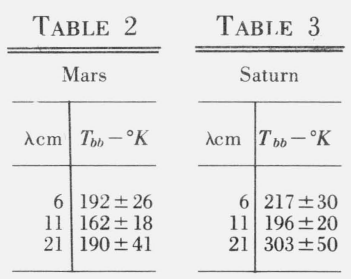

The difference between the infrared and radio temperatures can be interpreted as being due to the comparatively small diurnal temperature variations in the region below the Martian surface, surface where the 
longer wavelength emission may originate [Mayer, 1961]. Thus the radio measurements would refer to the average temperature over the entire surface of the planet, while infrared measurements made near opposition give the mean daytime temperature.

No evidence was found for the strong nonthermal emission reported by Davies at $21 \mathrm{~cm}$ [1964].

Saturn-The equivalent blackbody temperature of Saturn measured at 6,11 , and $21 \mathrm{~cm}$ is shown in table 3 . When compared to the values found near $3 \mathrm{~cm}-106$ $\pm 21{ }^{\circ} \mathrm{K}$ [Cook, Cross, Bair, and Arnold, 1960] and at infrared wavelengths $93 \pm 3^{\circ} \mathrm{K}$ [Low, 1964] they show an increase in the apparent temperature with increasing wavelength.

An attempt was also made to confirm the large polarization reported by the Naval Research Laboratory at $9.4 \mathrm{~cm}$ [Rose, Bologna, and Sloanaker, 1963]. No linear polarization at $11 \mathrm{~cm}$ was found greater than a few percent in agreement with the earlier results obtained at CSIRO by Davies, Beard, and Cooper [1964].

The absence of a significant amount of linear polarization suggests that the radio emission from Saturn is primarily of thermal origin and that the observed increase in temperature at the longer wavelengths is probably due to the higher temperature in the lower regions of Saturn's atmosphere. It is also possible that at the longer wavelengths the rings may contribute to the observed radio emission.

Uranus-Radio emission from Uranus was detected at $11 \mathrm{~cm}$ by alternately pointing the antenna toward the planet and then toward one or more reference positions located somewhat more than one beam width away. The receiver output was integrated for $100 \mathrm{sec}$ in each position, and the apparent antenna temperature was determined from the difference between the "on" and "off" positions. A total of about $4 \mathrm{hr}$ of useful integration time was obtained in each of the two positions. As the antenna temperature of Uranus is well below the confusion level of the $210-\mathrm{ft}$ telescope, the "on-off" sequence was repeated on a subsequent night when the planet had moved out of the beam, and the difference between the two sets of measurements was used to determine the flux density of Uranus.

The measured flux density was found to be $(8 \pm 3.5)$ $\times 10^{-29} \mathrm{~W} / \mathrm{m}^{2} / \mathrm{cps}$ corresponding to an equivalent blackbody temperature of $130 \pm 40{ }^{\circ} \mathrm{K}$. This is in reasonable agreement with the temperature expected from solar heating.
Part of this work was performed while the author held a National Science Foundation Postdoctoral Fellowship.

\section{References}

Cook, J. J., L. G. Cross, M. E. Bair, and C. B. Arnold (1960), Radio detection of the planet Saturn, Nature 188, No. 4748, 393-394.

Davies, R. D. (1964), Paper presented at the XII General Assembly of the International Astronomical Union, Hamburg, Germany, August 25-September 3.

Davies, R. D., M. Beard, and B. F. C. Cooper (1964), Observations of Saturn at 11.3 centimeters, Phys. Rev. Letters 13, No. 10 , 325-327.

Giordmaine, J. A., L. E. Alsop, C. H. Townes, and C. H. Mayer (1959), Observations of Jupiter and Mars at 3-cm wavelength, Astron. J. 64, No. 8, 332

Kellermann, K. I. (1965), 11-cm Observations of the temperature of Mercury, Nature 205, No. 4976, 1091-1092.

Low, F. J. (1964), Infrared brightness temperature of Saturn, Astron. J. 69, 550-551.

Mayer, C. H. (1961), Radio emission of the moon and planets, The Solar System, III, Planets and Satellites, ed. G. P. Kuiper and B. M. Middlehurst pp. 442-472, (Univ. of Chicago Press, Chicago).

Pettit, E. (1961), Planetary temperature measurement, The Solar System, III, Planets and Satellites, ed. G. P. Kuiper and B. M. Middlehurst, pp. 400-441 (Univ. of Chicago Press, Chicago).

Pettengill, G. H., and R. B. Dyce, (1965), Spring URSI meeting, Washington, D.C.

Rose, W. K., J. M., Bologna, and R. M. Sloanaker (1963), Linear polarization of the $3200-\mathrm{Mc} / \mathrm{sec}$ radiation from Saturn, Phys. Rev. Letters 10, No. 4, 123-125.

Discussion Following K. I. Kellermann's paper

F. D. Drake: It is both encouraging and pleasant to see the excellent agreement now being achieved by the planetary observers. With regard to Mars, during the 1963 opposition, we observed at $10-\mathrm{cm}$ a blackbody disk temperature of $177 \pm 17^{\circ} \mathrm{K}$, in excellent agreement with results reported here. I wonder if Dr. Sagan can explain the lower values of brightness temperature observed with Venus at the longer wavelengths.

C. Sagan: Unless you introduce inhomogeneities into the atmosphere, I do not see a way to explain these observations. Where was Mars during your observations?

K. I. Kellermann: The measurements were made in March 1965 near the ime of opposition.

(Paper 69D12-595) 